\title{
EXISTENCE OF A SINGULAR PROJECTIVE VARIETY WITH AN ARBITRARY SET OF CHARACTERISTIC NUMBERS
}

\author{
A. Y. BURYAK
}

It is known that Chern characteristic numbers of compact complex manifolds cannot have arbitrary values. They satisfy certain divisability conditions. For example (see, e.g., [5])

$$
\begin{array}{r}
2 \mid\left\langle c_{1}(X),[X]\right\rangle, \text { for } \operatorname{dim} X=1, \\
12 \mid\left\langle c_{1}^{2}(X)+c_{2}(X),[X]\right\rangle, \text { for } \operatorname{dim} X=2, \\
24 \mid\left\langle c_{1}(X) c_{2}(X),[X]\right\rangle, \text { for } \operatorname{dim} X=3 .
\end{array}
$$

W. Ebeling and S. M. Gusein-Zade ([1]) offer a definition of characteristic numbers of singular compact complex analytic varieties. For an $n$-dimensional singular analytic variety $X$, let $\nu: \widehat{X} \rightarrow X$ be its Nash transform and let $\widehat{T} X$ be the tautological bundle over $\widehat{X}$ (see, e.g, [1]). If $X$ is embedded into a smooth complex analytic manifold $M$, then over the nonsingular part $X_{\text {reg }}$ of $X$ there is a section of $G r_{n}(T M)$ given by the tangent space to $X$. The Nash transform $\widehat{X}$ is the closure in $G_{n}(T M)$ of the image of this section. The bundle $\widehat{T} X$ is the restriction to $\widehat{X}$ of the tautological bundle over $G r_{n}(T M)$. Let the variety $X$ be compact. For a partition $I=i_{1}, \ldots, i_{r}, i_{1}+\ldots+i_{r}=$ $n$ of $n$ the corresponding characteristic number $c_{I}[X]$ of the variety $X$ is defined by

$$
c_{I}[X]:=\left\langle c_{i_{1}}(\widehat{T} X) c_{i_{2}}(\widehat{T} X) \cdots c_{i_{r}}(\widehat{T} X),[\widehat{X}]\right\rangle,
$$

where $[\widehat{X}]$ is the fundamental class of the variety $\widehat{X}$. Let $\bar{c}[X]$ be the vector $\left(c_{I}[X]\right) \in$ $\mathbb{Z}^{p(n)}$, where $p(n)$ is the number of partitions of $n$.

Theorem. For any vector $\bar{v} \in \mathbb{Z}^{p(n)}$ there exists a projective variety $X$ of dimension $n$ such that $\bar{c}[X]=\bar{v}$.

Let $V$ be an algebraic variety. R. MacPherson ([6]) defined the local Euler obstruction $E u_{p}(V)$ of the variety $V$ at a point $p$. He proved that it is a constructible function on the variety $V$. Denote this function by $E u(X)$. The notion of the integral with respect to the Euler characteristic was defined in [9]. The proof of the Theorem will use the following fact.

Lemma 1. Let $X$ be a compact algebraic variety of dimension $n$; then $c_{n}[X]$ is equal to the following integral with respect to the Euler characteristic

$$
c_{n}[X]=\int_{X} E u(X) d \chi .
$$

Received by the editors October 29, 2009.

The author is partially supported by the grants RFBR-07-01-00593, NSh-709.2008.1 and the Vidi grant of NWO. 
Proof. For any constructible function $\alpha$ on the variety $X$ R. MacPherson ([6]) defined an element $c_{*}(\alpha) \in H_{*}(X)$. From his construction it follows that

$$
c_{n}[X]=\int_{X} c_{*}(E u(X)),
$$

where the integral means the degree of the class $c_{*}(E u(X))$. L. Ernström ([4]) proved that for any constructible function $\alpha$ on a variety $X$

$$
\int_{X} \alpha d \chi=\int_{X} c_{*}(\alpha) .
$$

Lemma 1 follows from these two formulas.

Proof of Theorem. We need some combinations of characteristic numbers (see, e.g., [7]). Define two monomials in $t_{1}, \ldots, t_{k}$ to be equivalent if some permutation of $t_{1}, \ldots, t_{k}$ transforms one into the other. Define $\sum t_{1}^{i_{1}} \cdots t_{r}^{i_{r}}$ to be the sum of all monomials in $t_{1}, \cdots, t_{k}$ equivalent to $t_{1}^{i_{1}} \cdots t_{r}^{i_{r}}$. For any partition $I=i_{1}, \ldots, i_{r}$ of $n$, define a polynomial $s_{I}$ in $n$ variables as follows. For $k \geq n$ elementary symmetric functions $\sigma_{1}, \ldots, \sigma_{n}$ of $t_{1}, \ldots, t_{k}$ are algebraically independent. Let $s_{I}$ be the unique polynomial satisfying

$$
s_{I}\left(\sigma_{1}, \ldots, \sigma_{n}\right)=\sum t_{1}^{i_{1}} \cdots t_{r}^{i_{r}} .
$$

This polynomial does not depend on $k$. Let $F$ be a complex vector bundle over a topological space $Y$. For a partition $I$ of $n$ the cohomology class $s_{I}\left(c_{1}(F), \ldots, c_{k}(F)\right) \in$ $H^{2 n}(Y)$ will be denoted by $s_{I}(F)$. For a compact analytic variety $X$ of dimension $n$ and a partition $I$ of $n$ let the number $s_{I}[X]$ be defined by

$$
s_{I}[X]:=\left\langle s_{I}(\widehat{T} X),[\widehat{X}]\right\rangle .
$$

Let $\bar{s}[X]$ be the vector $\left(s_{I}[X]\right) \in \mathbb{Z}^{p(n)}$. We have the following relationship between the vectors $\bar{c}[X]$ and $\bar{s}[X]$ (see, e.g., [7]). There exists a $p(n) \times p(n)$ matrix $A$ with integer coefficients and $\operatorname{det}(A)= \pm 1$ such that, for any compact analytic variety $X$ of dimension $n$, one has $\bar{c}[X]=A \bar{s}[X]$. Hence it is sufficient to prove that for any vector $\bar{v} \in \mathbb{Z}^{p(n)}$ there exists a projective variety $X$ such that $\bar{s}[X]=\bar{v}$.

For two complex bundles $F, F^{\prime}$ the characteristic class $s_{I}\left(F \oplus F^{\prime}\right)$ is equal to

$$
s_{I}\left(F \oplus F^{\prime}\right)=\sum_{J K=I} s_{J}(F) s_{K}\left(F^{\prime}\right),
$$

where the sum is over all partitions $J$ and $K$ with union $J K$ equal to $I([7])$.

Let $X_{1}, X_{2}$ be two compact analytic varieties and $\nu_{1}: \widehat{X_{1}} \rightarrow X_{1}, \nu_{2}: \widehat{X_{2}} \rightarrow X_{2}$ be their Nash transforms. It is clear that the map $\left(\nu_{1}, \nu_{2}\right): \widehat{X}_{1} \times \widehat{X}_{2} \rightarrow X_{1} \times X_{2}$ is the Nash transform of $X_{1} \times X_{2}$. Let $p_{1,2}: \widehat{X_{1}} \times \widehat{X_{2}} \rightarrow \widehat{X_{1,2}}$ be projections; then $\widehat{T}\left(X_{1} \times X_{2}\right)=p_{1}^{*} \widehat{T} X_{1} \oplus p_{2}^{*} \widehat{T} X_{2}$. Let $n_{1}$ and $n_{2}$ be the dimensions of $X_{1}$ and $X_{2}$. Let $I$ be a partition of $n_{1}+n_{2}$. From (1) it follows that

$$
s_{I}\left[X_{1} \times X_{2}\right]=\sum_{\substack{J K=I \\|J|=n_{1} \\|K|=n_{2}}} s_{J}\left[X_{1}\right] s_{K}\left[X_{2}\right] .
$$


Lemma 2. For any $i \geq 1$ there exist projective varieties $K_{+}^{i}$ and $K_{-}^{i}$ of dimension $i$ such that $s_{i}\left[K_{ \pm}^{i}\right]= \pm 1$.

We shall prove Lemma 2 later. Before that we shall deduce the statement of the Theorem from Lemma 2 . Let $J=j_{1}, \ldots, j_{q}$ be a partition of $n$. Let

$$
\begin{aligned}
& K_{+}^{J}=K_{+}^{j_{1}} \times K_{+}^{j_{2}} \times \cdots \times K_{+}^{j_{q}}, \\
& K_{-}^{J}=K_{-}^{j_{1}} \times K_{+}^{j_{2}} \times \cdots \times K_{+}^{j_{q}} .
\end{aligned}
$$

From (2) it follows that

$$
s_{I}\left[K_{ \pm}^{J}\right]=\sum_{\substack{I_{1} \cdots I_{q}=I \\\left|I_{l}\right|=j_{l}}} s_{I_{1}}\left[K_{ \pm}^{j_{1}}\right] s_{I_{2}}\left[K_{+}^{j_{2}}\right] \cdots s_{I_{q}}\left[K_{+}^{j_{q}}\right] .
$$

A refinement of a partition $J$ means any partition which can be written as a union $J_{1} \cdots J_{q}$ where each $J_{l}$ is a partition of $j_{l}$. Consider the lexicographical order on partitions of $n$. It is obvious that if $I$ is a refinement of $J$ then $I \leq J$. We see that the characteristic number $s_{I}\left[K_{ \pm}^{J}\right]$ is zero unless the partition $I$ is a refinement of $J$, hence $s_{I}\left[K_{ \pm}^{J}\right]=0$, if $I>J$. We have $s_{I}\left[K_{ \pm}^{I}\right]= \pm 1$. Now it is clear that the vectors $\bar{s}\left[K_{ \pm}^{J}\right]$ generate the whole lattice $\mathbb{Z}^{p(n)}$ as a semigroup. This finishes the proof of the theorem.

Proof of Lemma 2. It is known that, for any smooth compact algebraic variety $W$ of dimension $n$, there exists a smooth compact algebraic variety $V$ of dimension $n$ such that for any partition $I$ of the number $n$ we have $c_{I}[V]=-c_{I}[W]$ (see e.g. [8]). Denote the variety $V$ by $-W$. We have (see e.g. [7])

$$
s_{n}\left[\mathbb{C P}^{n}\right]=n+1 .
$$

We see that existence of a variety $K_{-}^{n}$ immediately follows from existence of a variety $K_{+}^{n}$ because $s_{n}\left[\left(-\mathbb{C P}^{n}\right)+n K_{+}^{n}\right]=-1$. We also see that it is sufficient to construct a projective variety $\widetilde{K}_{+}^{n}$ such that $s_{n}\left[\widetilde{K}_{+}^{n}\right] \equiv 1 \bmod n+1$.

Let $n=1$. Let $\widetilde{K}_{+}^{1}$ be the closure in $\mathbb{C P}^{2}$ of the semicubical parabola $\left\{x^{2}-y^{3}=\right.$ $0\} \subset \mathbb{C}^{2}$. From Lemma 1 and properties of the local Euler obstruction ([6]) it follows that $s_{1}\left[\widetilde{K}_{+}^{1}\right]=c_{1}\left[\widetilde{K}_{+}^{1}\right]=3 \equiv 1 \bmod 2$.

Let us construct varieties $\widetilde{K}_{+}^{n}$ for any $n \geq 2$. For a smooth subvariety $X \subset \mathbb{C P}^{N-1}$ of dimension $n-1$, let $C X \subset \mathbb{C P}^{N}$ be the cone over $X$. Let $h \in H^{2}\left(\mathbb{C P}^{N-1}\right)$ be the hyperplane class.

Lemma 3. Suppose the element $\left.h\right|_{X} \in H^{2}(X)$ is divisible by $d$; then

$$
s_{n}[C X] \equiv n s_{n-1}[X] \bmod d .
$$

Proof. Let $\mathbb{F}_{i_{1}, \ldots, i_{s}}$ be the variety consisting of flags $\left(V^{i_{1}}, \ldots, V^{i_{s-1}}\right)$ with $V^{i_{1}} \subset \cdots \subset$ $V^{i_{s-1}} \subset \mathbb{C}^{i_{s}}$ and $\operatorname{dim} V^{i_{k}}=i_{k}$. Denote by $D_{i_{k}}$ the tautological bundle over $\mathbb{F}_{i_{1}, \ldots, i_{s}}$. Let $p$ be a point of $\mathbb{C P}^{N}$ and let $V \subset T_{p} \mathbb{C P}^{N}$ be a $d$-dimensional subspace. Denote by $g(V)$ the unique $d$-dimensional projective subspace of $\mathbb{C P}^{N}$ such that $p \in g(V)$ and $T_{p}(g(V))=V$. Let $G \subset \mathbb{C P}^{N}$ be a $d$-dimensional projective subspace. By $k(G)$ 
denote the associated $(d+1)$-dimensional vector subspace of $\mathbb{C}^{N+1}$. Let $Y \subset \mathbb{C P}^{N}$ be an $n$-dimensional subvariety. Consider the map

$$
\sigma: Y_{\text {reg }} \rightarrow \mathbb{F}_{1, n+1, N+1}, Y_{\text {reg }} \ni p \mapsto\left(k(p), k\left(g\left(T_{p} Y_{\text {reg }}\right)\right)\right) \in \mathbb{F}_{1, n+1, N+1} .
$$

By definition the closure $\overline{\sigma\left(Y_{\text {reg }}\right)}$ is the Nash transform of $Y$. The bundle $\widehat{T} Y$ is isomorphic to $\left.\operatorname{Hom}\left(D_{1},\left(D_{n+1} / D_{1}\right)\right)\right|_{\widehat{Y}}$.

Let $\widehat{X} \subset \mathbb{F}_{1, n, N}$ and $\widehat{C X} \subset \mathbb{F}_{1, n+1, N+1}$ be the Nash transforms of $X$ and $C X$ respectively. Consider the diagram

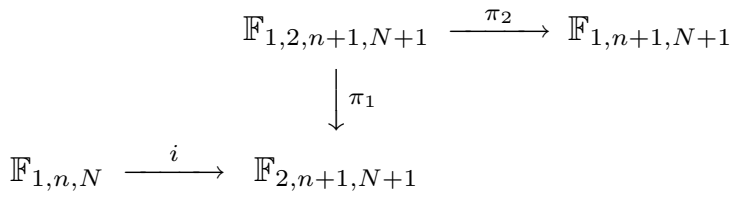

where $\pi_{1}, \pi_{2}$ are the natural projections and the map $i$ is defined by

$$
i:\left(V^{1}, V^{n}\right) \mapsto\left(V^{1} \oplus k(O), V^{n} \oplus k(O)\right),
$$

where $O \in \mathbb{C P}^{N}$ is the vertex of the cone $C X$. Obviously the map $i$ is injective. Let $Y=\pi_{1}^{-1}(i(\widehat{X}))$.

Lemma 4. The image of $Y$ under the map $\pi_{2}$ is $\widehat{C X}$. The $\operatorname{map} \pi_{2}: Y \rightarrow \widehat{C X}$ is birational.

Proof. Denote by $\overline{p q}$ the line, which goes through two different points $p, q \in \mathbb{C P}^{N}$. From the definition of the variety $Y$ it follows that

$$
\begin{gathered}
Y=\left\{\left(L, k(q) \oplus k(O), k\left(g\left(T_{q} X\right)\right) \oplus k(O)\right) \in \mathbb{F}_{1,2, n+1, N+1} \mid\right. \\
q \in X, L \subset k(q) \oplus k(O)\}= \\
=\left\{\left(k(p), k(q) \oplus k(O), k\left(g\left(T_{q} X\right)\right) \oplus k(O)\right) \in \mathbb{F}_{1,2, n+1, N+1} \mid\right. \\
q \in X, p \in C X, p \in \overline{q O}\} .
\end{gathered}
$$

Note that, if $p \neq O$, then $q$ is uniquely determined by $p$. Denote by $Y^{\prime}$ the subset of triples from (4) such that $p \neq O$.

It is clear that for any point $p \in C X \backslash\{O\}$ we have

$$
k\left(g\left(T_{p} C X\right)\right)=k\left(g\left(T_{\overline{p O} \cap X} X\right)\right) \oplus k(O) .
$$

We see that for any element $\left(V^{1}, V^{n+1}\right) \in \widehat{C X} \subset \mathbb{F}_{1, n+1, N+1}$ there exist points $p \in C X$ and $q \in X$ such that $p \in \overline{q O}$ and

$$
V^{1}=k(p), V^{n+1}=k\left(g\left(T_{q} X\right)\right) \oplus k(O) .
$$

Note that a point $q$ is not uniquely determined by the element $\left(V^{1}, V^{n+1}\right)$.

The map $\pi_{2}$ just forgets the second element of the triple from (4) and it is clear that we obtain the pair $\left(V^{1}, V^{n+1}\right)$ from (5). This completes the proof of the first part of the lemma.

Let $\widehat{C X}^{\prime}=\left\{\left(V^{1}, V^{n+1}\right) \in \widehat{C X} \mid V^{1} \neq k(O)\right\}$. Note that if $\left(V^{1}, V^{n+1}\right) \in \widehat{C X}^{\prime}$ then a point $q$ from (5) is uniquely determined and $q=\overline{p O} \cap X$. Now it is clear that the map $\pi_{2}$ sends $Y^{\prime}$ isomorphically onto $\widehat{C X}^{\prime}$. This concludes the proof of the second part of the lemma. 
Denote by $\widetilde{D}_{i}$ the tautological bundles over $\mathbb{F}_{2, n+1, N+1}, \mathbb{F}_{1,2, n+1, N+1}, \mathbb{F}_{1, n+1, N+1}$. Denote by $D_{i}$ the tautological bundles over $\mathbb{F}_{1, n, N}$. We have

$$
\begin{gathered}
s_{n}[C X]=\left\langle s_{n}(\widehat{T}(C X)),[\widehat{C X}]\right\rangle=\left\langle s_{n}\left(\widetilde{D}_{1}^{*} \otimes\left(\widetilde{D}_{n+1} / \widetilde{D}_{1}\right)\right),[\widehat{C X}]\right\rangle \stackrel{\text { lemma } 4}{=} \\
=\left\langle s_{n}\left(\widetilde{D}_{1}^{*} \otimes\left(\widetilde{D}_{n+1} / \widetilde{D}_{1}\right)\right),[Y]\right\rangle .
\end{gathered}
$$

The map $\pi_{1}: \mathbb{F}_{1,2, n+1, N+1} \rightarrow \mathbb{F}_{2, n+1, N+1}$ is the projectivization of the bundle $\widetilde{D}_{2}$ over $\mathbb{F}_{2, n+1, N+1}$. We have that $i^{*} \widetilde{D}_{2} \cong D_{1} \oplus \mathbb{C}$ and $i^{*} \widetilde{D}_{n+1} \cong D_{n} \oplus \mathbb{C}$. We see that the variety $Y$ is the total space $\mathbb{P}\left(D_{1} \oplus \mathbb{C}\right)$ of the projectivization of the bundle $D_{1} \oplus \mathbb{C}$ over $\widehat{X}$. By $\tau$ we denote the tautological bundle over $\mathbb{P}\left(D_{1} \oplus \mathbb{C}\right)$. It is clear that $\tau=\left.\widetilde{D}_{1}\right|_{Y}$. Therefore we have

$$
\left\langle s_{n}\left(\widetilde{D}_{1}^{*} \otimes\left(\widetilde{D}_{n+1} / \widetilde{D}_{1}\right)\right),[Y]\right\rangle=\left\langle s_{n}\left(\tau^{*} \otimes\left(\left(D_{n} \oplus \mathbb{C}\right) / \tau\right)\right),\left[\mathbb{P}\left(D_{1} \oplus \mathbb{C}\right)\right]\right\rangle .
$$

Moreover

$$
\begin{gathered}
s_{n}\left(\tau^{*} \otimes\left(\left(D_{n} \oplus \mathbb{C}\right) / \tau\right)\right)=s_{n}\left(\tau^{*} \otimes\left(\left(D_{n} / D_{1}\right) \oplus D_{1} \oplus \mathbb{C}\right)\right)= \\
=s_{n}\left(\tau^{*} \otimes\left(D_{n} / D_{1}\right)\right)+s_{n}\left(\tau^{*} \otimes D_{1}\right)+s_{n}\left(\tau^{*}\right)= \\
=s_{n}\left(\tau^{*} \otimes D_{1} \otimes\left(D_{1}^{*} \otimes\left(D_{n} / D_{1}\right)\right)\right)+s_{n}\left(\tau^{*} \otimes D_{1}\right)+s_{n}\left(\tau^{*}\right)= \\
=s_{n}\left(\tau^{*} \otimes D_{1} \otimes \widehat{T} X\right)+s_{n}\left(\tau^{*} \otimes D_{1}\right)+s_{n}\left(\tau^{*}\right) .
\end{gathered}
$$

Let $c_{1}\left(\tau^{*}\right)=u \in H^{2}\left(\mathbb{P}\left(D_{1} \oplus \mathbb{C}\right)\right)$. We have $u^{2}=u h$. Therefore from the assumption of the lemma it follows that for any $k \geq 2$ the element $u^{k} \in H^{2 k}\left(\mathbb{P}\left(D_{1} \oplus \mathbb{C}\right)\right)$ is divisible by $d$. Hence we have

$$
\begin{aligned}
\left\langle s_{n}\left(\tau^{*} \otimes D_{1}\right),\left[\mathbb{P}\left(D_{1} \oplus \mathbb{C}\right)\right]\right\rangle & =\left\langle(u-h)^{n},\left[\mathbb{P}\left(D_{1} \oplus \mathbb{C}\right)\right]\right\rangle \equiv 0 \quad \bmod d \\
\left\langle s_{n}\left(\tau^{*}\right),\left[\mathbb{P}\left(D_{1} \oplus \mathbb{C}\right)\right]\right\rangle & =\left\langle u^{n},\left[\mathbb{P}\left(D_{1} \oplus \mathbb{C}\right)\right]\right\rangle \equiv 0 \quad \bmod d .
\end{aligned}
$$

Let $x_{1}, \ldots, x_{n-1}$ be Chern roots of the bundle $\widehat{T} X$. Then $x_{1}+u-h, \ldots, x_{n-1}+u-h$ are Chern roots of the bundle $\tau^{*} \otimes D_{1} \otimes \widehat{T} X$. Hence

$$
\begin{gathered}
\left\langle s_{n}\left(\tau^{*} \otimes D_{1} \otimes \widehat{T} X\right),\left[\mathbb{P}\left(D_{1} \oplus \mathbb{C}\right)\right]\right\rangle= \\
=\left\langle\sum_{i=1}^{n-1}\left(x_{i}+u-h\right)^{n},\left[\mathbb{P}\left(D_{1} \oplus \mathbb{C}\right)\right]\right\rangle \equiv\left\langle\sum_{i=1}^{n-1}\left(x_{i}+u\right)^{n},\left[\mathbb{P}\left(D_{1} \oplus \mathbb{C}\right)\right]\right\rangle \equiv \\
\equiv\left\langle\sum_{i=1}^{n-1} x_{i}^{n}+n u \sum_{i=1}^{n-1} x_{i}^{n-1},\left[\mathbb{P}\left(D_{1} \oplus \mathbb{C}\right)\right]\right\rangle \bmod d .
\end{gathered}
$$

The class $\sum_{i=1}^{n-1} x_{i}^{n} \in H^{2 n}(\widehat{X})$ is equal to zero because $\operatorname{dim}_{\mathbb{R}} \widehat{X}=2 n-2$. Therefore

$$
\begin{gathered}
\left\langle\sum_{i=1}^{n-1} x_{i}^{n}+n u \sum_{i=1}^{n-1} x_{i}^{n-1},\left[\mathbb{P}\left(D_{1} \oplus \mathbb{C}\right)\right]\right\rangle=\left\langle n u s_{n-1}(\widehat{T} X),\left[\mathbb{P}\left(D_{1} \oplus \mathbb{C}\right)\right]\right\rangle= \\
=n\left\langle\left(\pi_{1 *} u\right) s_{n-1}(\widehat{T} X),[\widehat{X}]\right\rangle=n\left\langle s_{n-1}(\widehat{T} X),[\widehat{X}]\right\rangle=n s_{n-1}[X] .
\end{gathered}
$$

This completes the proof of Lemma 3. 
Let $X=\mathbb{C P}^{n-1} \hookrightarrow \mathbb{C P}\left(\begin{array}{c}2 n \\ n-1\end{array}\right)-1$ be the image of the Veronese embedding of degree $n+1$. Let $\widetilde{K}_{+}^{n}=C X$. From $(3)$ and Lemma 3 it follows that $s_{n}\left[\widetilde{K}_{+}^{n}\right] \equiv n^{2} \equiv 1$ $\bmod n+1$. This concludes the proof of Lemma 2 .

Remark. It seems to be interesting to construct a cobordism theory of singular varieties associated to this notion of characteristic numbers.

\section{Acknowledgements}

The author is grateful to professor S. M. Gusein-Zade for suggesting the problem and for constant attention to this work.

\section{References}

[1] W. Ebeling, S. M. Gusein-Zade. Chern obstructions for collections of 1-forms on singular varieties. Singularity Theory, 557-564, World Sci. Publ., Hackensack, NJ, 2007.

[2] W. Ebeling, S. M. Gusein-Zade. Indices of collections of 1-forms. Singularities in geometry and topology, 629-639, World Sci. Publ., Hackensack, NJ, 2007.

[3] W. Ebeling, S. M. Gusein-Zade. Indices of vector fields or 1-forms and characteristic numbers. Bull. London Math. Soc. 37 (2005), no. 5, 747-754.

[4] L. Ernström. Topological Radon transforms and the local Euler obstruction. Duke Math. J. 76 (1994), no. 1, 1-21.

[5] F. Hirzebruch. Topological methods in algebraic geometry. Springer-Verlag, New York, 1966.

[6] R. MacPherson. Chern classes for singular algebraic varieties. Ann. of Math. (2) 100 (1974), $423-432$.

[7] Milnor J.W., Stasheff J.D. Characteristic classes. Annals of Mathematics Studies, No. 76. Princeton University Press, 1974.

[8] R. E. Stong. Notes on cobordism theory. Mathematical notes Princeton University Press, 1968.

[9] Viro, O. Ya. Some integral calculus based on Euler characteristic. Topology and geometryRohlin Seminar, 127-138, Lecture Notes in Math., 1346, Springer, Berlin, 1988.

Faculty of Mechanics and Mathematics, Moscow State University, 119991 Moscow, RUSSIA AND

Department of Mathematics, University of Amsterdam, P. O. Box 94248, 1090 GE AmsTERDAm, The Netherlands

E-mail address: buryaksh@mail.ru, a.y.buryak@uva.nl 